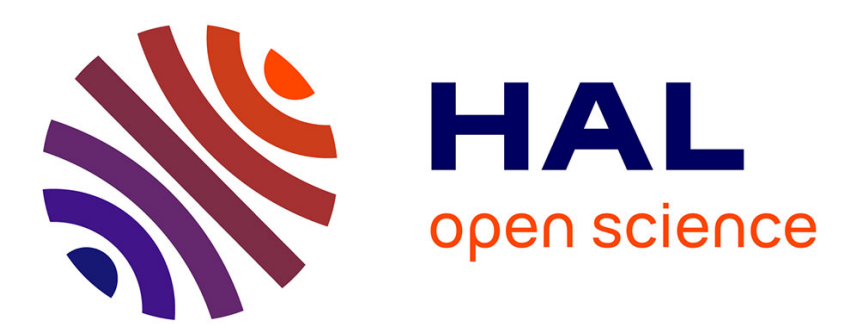

\title{
Multistep quadratic cascading in broadband optical parametric generation
}

Martin Levenius, Matteo Conforti, Fabio Baronio, Valdas Pasiskevicius, Fredrik Laurell, Costantino de Angelis, Katia Gallo

\section{To cite this version:}

Martin Levenius, Matteo Conforti, Fabio Baronio, Valdas Pasiskevicius, Fredrik Laurell, et al.. Multistep quadratic cascading in broadband optical parametric generation. Optics Letters, 2012, 37 (10), pp.1727. 10.1364/OL.37.001727 . hal-02394933

\section{HAL Id: hal-02394933 \\ https://hal.science/hal-02394933}

Submitted on 5 Dec 2019

HAL is a multi-disciplinary open access archive for the deposit and dissemination of scientific research documents, whether they are published or not. The documents may come from teaching and research institutions in France or abroad, or from public or private research centers.
L'archive ouverte pluridisciplinaire HAL, est destinée au dépôt et à la diffusion de documents scientifiques de niveau recherche, publiés ou non, émanant des établissements d'enseignement et de recherche français ou étrangers, des laboratoires publics ou privés. 


\title{
Multistep quadratic cascading in broadband optical parametric generation
}

\author{
Martin Levenius, ${ }^{1}$ Matteo Conforti, ${ }^{2}$ Fabio Baronio, ${ }^{2}$ Valdas Pasiskevicius, ${ }^{1}$ \\ Fredrik Laurell, ${ }^{1}$ Costantino De Angelis, ${ }^{2}$ and Katia Gallo ${ }^{1, *}$ \\ ${ }^{1}$ Department of Applied Physics, KTH - Royal Institute of Technology, Roslagstullsbacken 21, 10691 Stockholm, Sweden \\ ${ }^{2}$ CNISM and Department of Information Engineering, University of Brescia, via Branze 38, I-25123 Brescia, Italy \\ *Corresponding author: gallo@kth.se
}

Received Month X, XXXX; revised Month X, XXXX; accepted Month X, XXXX; posted Month X, XXXX (Doc. ID XXXXX); published Month X, XXXX

\begin{abstract}
We investigate theoretically and experimentally multistep parametric processes in broadband optical parametric generators based on periodically poled $1 \mathrm{~mol} \% \mathrm{MgO}$-doped stoichiometric $\mathrm{LiTaO}_{3}$. We demonstrate that parametric collateral processes may deplete or enhance spectral portions of the optical parametric generation output, depending on pump pulse duration. (C) 2011 Optical Society of America
\end{abstract}

OCIS Codes: 190.4410, 190.4360

A wide range of applications, encompassing ultrafast spectroscopy, high-harmonic and attosecond pulse generation, frequency combs and remote sensing, spur current research on broadband optical sources in the infrared [1-2]. Coherent ultra-broadband parametric gain suitable for ultrashort pulse generation and amplification can be afforded by quadratic frequency down-conversion in Quasi Phase-Matched (QPM) materials operated close to their zero group velocity dispersion point. Based on this principle, broadband optical parametric generation (OPG) has been achieved in periodically patterned GaAs [3], $\mathrm{KTiOPO}_{4}$ [4], $\mathrm{LiNbO}_{3}$ [5], and, recently, $\mathrm{LiTaO}_{3}$, with a record gain bandwidth of $180 \mathrm{THz}$ [6].

The spectral flatness of the parametric gain curves is a feature almost as important as the breadth of its spectral coverage. However, multistep $\chi^{(2)}$ processes triggered by broadband OPG, such as sum-frequency generation (SFG), often disrupt the flatness of gain profiles in QPM media [3-6].

In this Letter we investigate theoretically and experimentally the impact of multistep $\chi^{(2)}$ processes on broadband OPG in the pulsed regime. With reference to SFG, we show how quadratic cascading [7] can be used to engineer the OPG spectral profiles. Specifically, we demonstrate that the SFG-induced gain dips commonly observed in broadband OPG [3-6] can be turned into peaks by suitably choosing the pulse duration of the OPG pump. Experiments conducted in periodically poled $\mathrm{MgO}$ doped stoichiometric $\mathrm{LiTaO}_{3}$ (PPMgSLT) [6] provide evidence for theoretical predictions based on a model accounting rigorously for broadband OPG and multistep processes [8].

The multistep quadratic interactions which can affect ultra-broadband parametric generation in a periodically poled medium are schematically illustrated by Fig. 1a. In the $\chi^{(2)}$ material, the optical pump at $\lambda_{\mathrm{P}}$ excites parametric generation in the infrared region $\left(\lambda_{\mathrm{OPG}}\right)$ via first order QPM. High-order resonances of the QPM grating can simultaneously enable SFG between the pump and specific components ( $\lambda_{\mathrm{s}}$ in Fig. 1) within the broadband OPG spectrum. a)

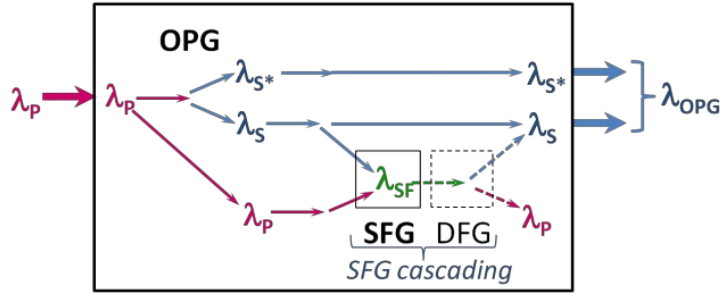

b)

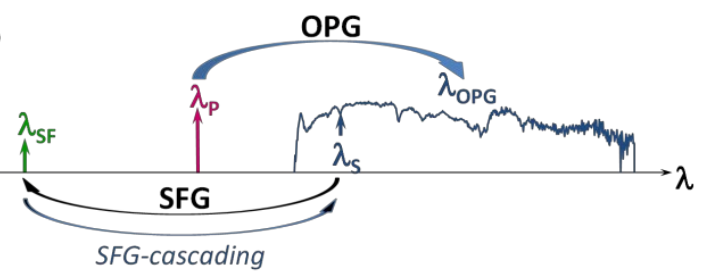

Fig. 1. a) Interaction paths of multistep $\chi^{(2)}$ processes triggered by broadband optical parametric generation (OPG) in a QPM medium. $\mathrm{SFG}=$ sum frequency generation, $\mathrm{DFG}=$ difference frequency generation. (b) Spectral signatures of OPG + SFG in the spectral domain (experimental data ref. [6]).

The SFG process $\left(1 / \lambda_{P}+1 / \lambda_{S} \rightarrow 1 / \lambda_{S F}\right)$ subtracts energy from the OPG band, carving a gain dip at $\lambda \mathrm{s}$, as highlighted on the spectrum of Fig. 1b. This is the situation typically encountered in the experiments [3-6].

The unwanted effects of parasitic SFG processes on the parametric gain could in principle be alleviated through more sophisticated QPM grating designs, yet this option becomes ever more challenging as the OPG spectra get wider. Alternatively, instead of trying to remove the QPM resonances responsible for upconversion, one could exploit $S F G$ cascading to re-route power into the OPG band via difference frequency generation (DFG, compare Fig. 1a).

In principle, according to the theory of quadratic cascading for non-degenerate frequency upconversion [9], the direction of energy flow in the SFG step is reversible (even at phase-matching) as long as depletion of one of the waves (either $\lambda_{\mathrm{S}}$ or $\lambda_{\mathrm{P}}$ ) is locally achieved in the device. In our configuration (Fig. 1a) the signal $\left(\lambda_{\mathrm{S}}\right)$ is generated by the OPG pump, therefore the control of $S F G$ cascading ultimately resides only in the latter (i.e. the input at $\lambda_{\mathrm{P}}$ ). 
To assess the impact of SFG cascading on broadband parametric generation, we performed a systematic numerical analysis based on a recently developed mathematical model [8], which can account rigorously for all the spectral components and multiple interactions of Fig.1. We explored the device response in the pulsed regime under experimentally viable conditions [6], referring to OPG in a $1 \mathrm{~cm}$-long PPMgSLT crystal, doped with $1 \mathrm{~mol} \% \mathrm{MgO}$, with a QPM grating with a period of $25 \mu \mathrm{m}$ and a duty cycle of $42.5 \%$, operated at a temperature $\mathrm{T}=80^{\circ} \mathrm{C}$ and pumped close its zerodispersion point $\left(\lambda_{\mathrm{P}}=860 \mathrm{~nm}\right)$ by Gaussian pulses at $1 \mathrm{kHz}$, with peak intensities in the range $1-10 \mathrm{GW} / \mathrm{cm}^{2}$ and pulse durations ranging between 0.5 and $30 \mathrm{ps}$.

The simulations employed the following quadratic nonlinear envelope equation [8]:

$$
\frac{d A}{d z}+i D A=-\frac{i \chi^{(2)} \omega_{0}^{2}}{4 \beta_{0} c^{2}}\left(1-\frac{i}{\omega_{0}} \frac{\partial}{\partial \tau}\right)\left[A^{2} e^{i \phi}+2|A|^{2} e^{-i \phi}\right]
$$

where $A(\mathrm{z}, \mathrm{t})$ is the broadband complex electric field envelope at a reference pulsation $\omega_{0}, \phi(z, t)=i \omega_{0} \tau-$ $i\left(\beta_{0}-k_{1} \omega_{0}\right) z, \beta_{0}=\operatorname{Re}\left[k\left(\omega_{0}\right)\right], k(\omega)=(\omega / c) \sqrt{1+\hat{\chi}(\omega)}$ being the propagation constant and $\hat{\chi}(\omega)$ the linear electric susceptibility. $D=\sum_{m=2}^{\infty} \frac{1}{m !} k_{m}\left(-i \frac{\partial}{\partial t}\right)^{m}$ is the dispersion operator, $k_{m}=\frac{\partial^{m} k}{\partial \omega^{m}}\left(\omega_{0}\right), \chi^{(2)}$ is the second order nonlinearity, $z$ the propagation coordinate, and $\tau=$ $t-k_{1} z$ the coordinate system, moving with the pump group velocity. The model refers to a plane-wave configuration, with no dependence on the transverse coordinates. Material dispersion is accounted for based on ref. [10].

We simulated OPG from a classical Gaussian pump and a semiclassical pulse shot noise of one photon per mode with random phase on each spectral discretization bin. The OPG response was statistically evaluated over the ensemble of multiple simulations, performed with different random noise seeds. We typically used an average of 30 realizations, which compares well with the experimental conditions described in what follows.

Fig. 2 illustrates key features of the calculated response, by considering OPG in PPMgSLT with a pump at $860 \mathrm{~nm}$. In each picture, the upper plot shows the broadband $\mathrm{OPG}$ spectrum (sweeping from 1 to $3.5 \mu \mathrm{m}$ ) at the output, while the contour plot illustrates its evolution in propagation (along z) within the PPMgSLT crystal. Fig. 2a and 2b concern two different pump pulse durations, namely 10 and $0.7 \mathrm{ps}$, and peak intensities, namely 7 and $15 \mathrm{GW} / \mathrm{cm}^{2}$, respectively.

Fig. 2a refers to the situation generally encountered in the experiments, where SFG induces localised dips in the gain spectra. In this specific case the dips, located at 1.4, $1.5,1.95$ and $2.2 \mu \mathrm{m}$, are the signatures of two distinct SFG processes occurring in the PPMgSLT device, namely:

- SFG between $\lambda_{\mathrm{P}}$ and $\lambda_{\mathrm{S} 1}=1.4 \mu \mathrm{m}$, generating a wave

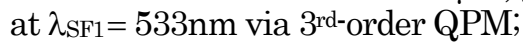

- SFG between $\lambda_{\mathrm{P}}$ and $\lambda_{\mathrm{S} 2}=1.95 \mu \mathrm{m}$, generating a wave at $\lambda_{\mathrm{SF} 2}=600 \mathrm{~nm}$ via $2^{\text {nd-order }} \mathrm{QPM}$.

The two SFG processes affect also the OPG gain at the conjugate wavelengths $\left[\lambda_{\mathrm{S}^{*}}=\lambda \mathrm{s} \cdot \lambda_{\mathrm{P}} /\left(\lambda_{\mathrm{S}}-\lambda \mathrm{P}\right)\right]$, located at $\lambda \mathrm{S}^{*}=2.2 \mu \mathrm{m}$ and $\lambda \mathrm{S}^{*}=1.5 \mu \mathrm{m}$, respectively.

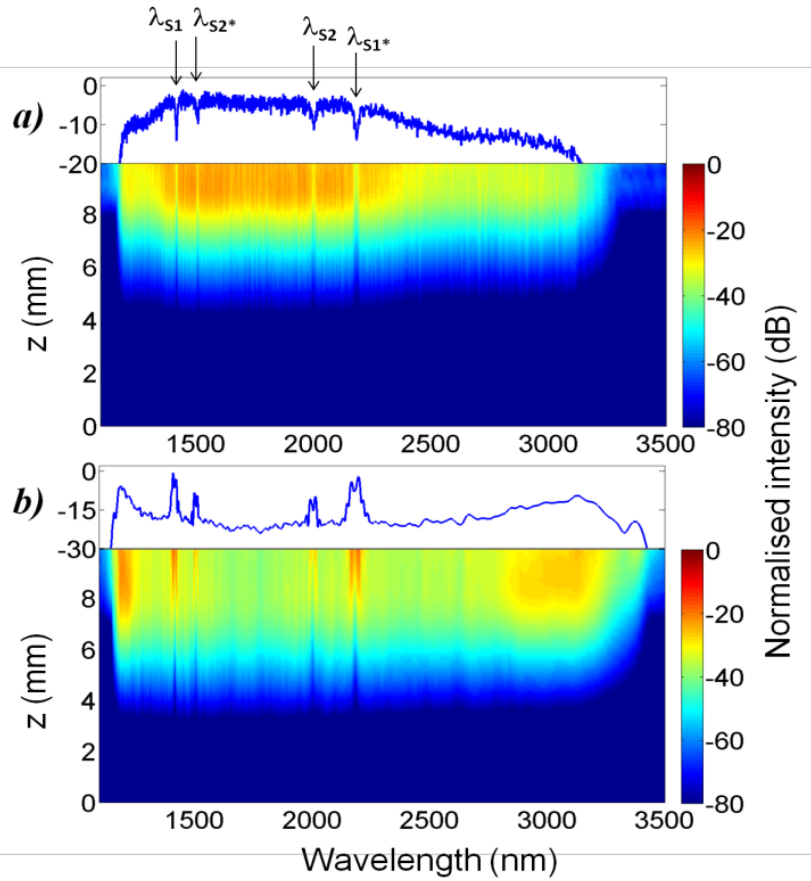

Fig. 2. Simulated OPG response of a 1cm-long PPMgSLT device in the presence of cascaded processes, with a Gaussian pump at $\lambda_{\mathrm{P}}=860 \mathrm{~nm}$. Input pulse durations: a) 10ps and b) 0.7ps. Peak intensities: a) $7 \mathrm{GW} / \mathrm{cm}^{2}$ and b) $15 \mathrm{GW} / \mathrm{cm}^{2}$. Output spectra (1D plots) and OPG signal evolution inside the device (contour plots). Numerical solutions of Eq. (1), averaged over 30 realizations.

Fig. 2b shows simulations for the same device of Fig. 2a, this time pumped with pulses of a much shorter duration (0.7ps). As apparent from the output spectrum, a gain enhancement now occurs at the very same spectral locations $\left(\lambda_{\mathrm{s} 1}, \lambda_{\mathrm{s} 2}, \lambda_{\mathrm{S} 1^{*}}, \lambda_{\mathrm{S} 2^{*}}\right)$ where $\mathrm{SFG}$ was carving dips in the gain curve of Fig. 2a. This happens as a consequence of the onset of SFG cascading, which brings power back into the OPG band by activating the DFG channel of Fig. 1a.

The simulation results point out to a crucial role played by pulse walk-off on the onset of SFG cascading. As the pump pulse duration is decreased, the walk-off among the spectral components involved in the SFG process $\left(\lambda_{\mathrm{P}}, \lambda_{\mathrm{SF}}\right.$ and $\lambda_{\mathrm{S}}$ ) increases. Accordingly, as these components lose their overlap upon propagation, the SF wave begins to back-convert its power to $\lambda_{\mathrm{P}}$ and $\lambda_{\mathrm{S}}$, yielding an enhancement of the OPG output. This agrees well with what would be expected for a single three-wave mixing interaction, where periodic up- and down- conversion takes place with a spatial period which depends on field intensities and temporal walk off [11].

The above intuitive explanation for the results of Fig. 2 is also consistent with the evolution of the interacting fields observed in the time domain, illustrated by Fig. 3. As seen in Fig. 3a, with the longer input pulses (10 ps), since the pump and the generated visible and infrared waves maintain their overlap, the temporal distribution of the total pulse exhibits no significant offset with respect to the input pump $(\mathrm{t}=0)$. This leads to efficient $\mathrm{SFG}$, hence depletion of the OPG outputs at $\lambda_{\mathrm{S} 1,2}$ and $\lambda_{\mathrm{s} 12^{*}}$ (Fig. 2a). On the other hand, with shorter pulses (0.7ps) the visible and infrared components generated in the device get 
away faster from the pump. This effect can be recognised in the time domain propagation plot of Fig. 3b, where a skewed trace (associated to components walking-off from the pump) clearly emerges for $z \geq 6 \mathrm{~mm}$. The progressive reduction of the temporal overlap among the spectral components involved in SFG locally alters the frequency conversion dynamics, starting to feed power back in the infrared region via DFG (Fig. 2b).
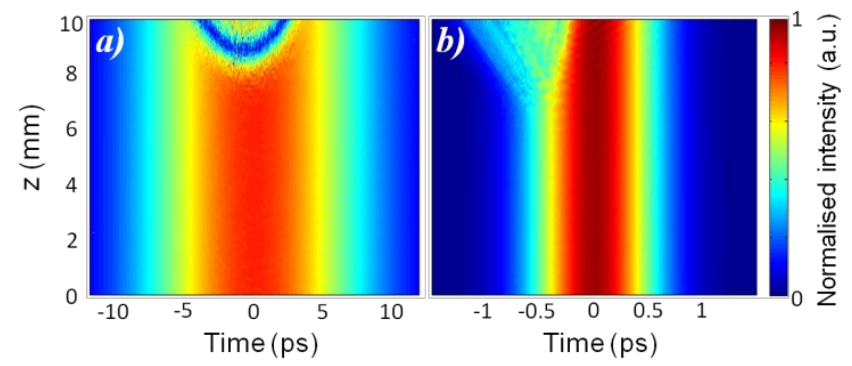

Fig. 3. Averaged total field evolution in the time domain, corresponding to the configurations of Fig. 2, namely: Gaussian pump pulses of a) 10 ps and b) $0.7 \mathrm{ps}$.

To confirm the above predictions, we then set to investigate the response of ultra-broadband $\mathrm{OPG}$ in PPMgSLT [6] under varying pumping conditions, seeking experimental evidence for $S F G$ cascading. The samples used for these experiments were $500 \mu \mathrm{m}$-thick, $1 \mathrm{~cm}$-long, z-cut substrates poled with a constant period of $25 \mu \mathrm{m}$.

The pump source was a tunable picosecond Ti:sapphire amplifier system, consisting of a $\mathrm{Nd}: \mathrm{YVO}_{4}$-pumped femtosecond source which seeded a regenerative chirped pulse amplifier, delivering micro-joule pulses at $1 \mathrm{kHz}$ with bandwidths of $2 \mathrm{~nm}$, tunable from 820 to $900 \mathrm{~nm}$. We varied the pump pulse durations by adjusting the group delay dispersion introduced by the final compressor stage of the amplifier. We used a $300 \mathrm{~mm}$ lens to loosely focus the pump beam to a radius of $100 \mu \mathrm{m}$ at the centre of the PPMgSLT grating. At the sample ends the radius increased only by $10 \%$, justifying the plane-wave approximation used in the model. The crystal was kept at $80^{\circ} \mathrm{C}$. At its output, after rejecting the residual pump with a dichroic filter, we recorded the signal spectra with a $0.55 \mathrm{~m}$ spectrometer (Horiba Jobin Yvon iHR550) sensitive up to $4.8 \mu \mathrm{m}$, averaging over 900 pulses.

Within the limits of our experimental setup, we systematically investigated the impact of input pulse durations and powers on the output OPG spectra. Fig. 4 summarises the key results, by comparing two measurements, taken for the same pump peak intensity $\left(6 \mathrm{GW} / \mathrm{cm}^{2}\right)$, but with two different input pulse durations, namely 6.1 and $1.7 \mathrm{ps}$.

A comparison with the spectral responses expected with long and short pump pulses, respectively (Fig. 2a-b), confirms the good agreement between our experimental observations (Fig. 4) and theory. The spectrum recorded with long pump pulses (6.1 ps, red curve in Fig. 4) bears the characteristic signatures of SFG, exhibiting a marked depletion of the OPG output at four signal wavelengths $(1.4,1.5,1.95$ and $2.2 \mu \mathrm{m}$, highlighted by the arrows), which match well the theoretical predictions (Fig. 2a). On the other hand, when pulse durations are reduced (1.7 ps, black curve in Fig. 4), the OPG output is locally enhanced at the same spectral locations where the gain reduction was apparent with long pulses, as predicted by theory (Fig. 2b), due to $S F G$ cascading.

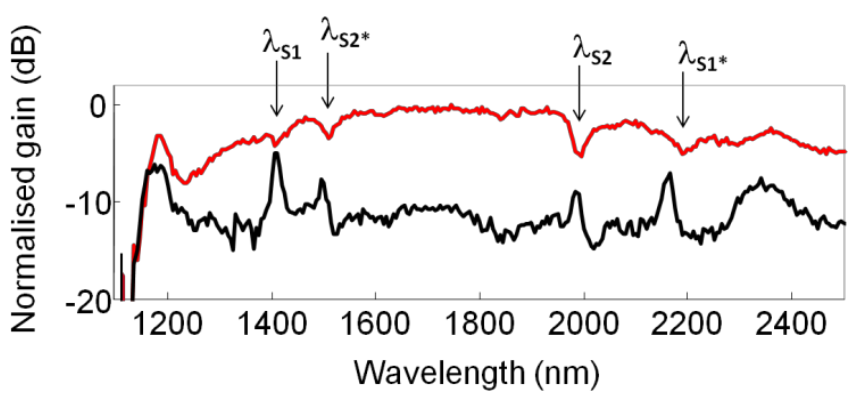

Fig. 4. OPG spectra measured with a pulsed pump at $860 \mathrm{~nm}$, with Gaussian pulse durations of $6.1 \mathrm{ps}$ (red line) and $1.7 \mathrm{ps}$ (black line), at $6 \mathrm{GW} / \mathrm{cm}^{2}$ in a $1 \mathrm{~cm}$ PPMgSLT crystal.

In conclusion, we studied the interplay of multistep quadratic processes with broadband OPG in the pulsed regime. We demonstrated the possibility to counteract the impact of SFG on the parametric gain by triggering SFGcascading to channel energy back into the OPG band. Simulations and experiments in PPMgSLT show how the energy flow in the SFG step can be reversed by reducing the pump pulse duration so to drive walk-off initiated down-conversion of the SF wave, turning the gain dips typically observed during broadband OPG in QPM media into peaks. Simulations predict also the possibility to access an intermediate regime, without dips nor peaks, with significant improvements in the flatness of the OPG spectra, by fine-tuning the input pulse duration in relation to the sample length. Ultimately, these results point out to new possibilities to engineer all-optically the response of broadband parametric sources and amplifiers in QPM materials.

The present research is supported at KTH by the Swedish Research Council (VR fellowship 6222010 526, VR grant 62120114040 and ADOPT Linnée Centre) and in Brescia by MIUR (PRIN 2009P3K72Z) and Fondazione CARIPLO (2010-0595).

\section{References}

1. G. Cerullo and S. De Silvestri, Rev. Sci. Instrum. 74, 1 (2003).

2. R. Butkus, R. Danielius, A. Dubietis, A. Piskarskas, and A. Stabinis, Appl. Phys. B 79, 693 (2004).

3. P. Kuo, K. Vodopyanov, M. Fejer, D. Simanovskii, X. Yu, J. Harris, D. Bliss, and D. Weyburne, Opt. Lett. 31, 71 (2006).

4. M. Tiihonen, V. Pasiskevicius, A. Fragemann, C. Canalias, and F. Laurell, Appl. Phys. B 85, 73 (2006).

5. O. Prakash, H. H. Lim, B. J. Kim, K. Pandiyan, M. Cha, and B. Rhee, Appl. Phys. B 92, 535 (2008).

6. M. Levenius, V. Pasiskevicius, F. Laurell, and K. Gallo, Opt. Express 19, 4121 (2011).

7. G.I. Stegeman, Quantum Semiclass. Opt. 9, 139 (1997).

8. M. Conforti, F. Baronio, C. De Angelis, Phys. Rev. A 81, 053841 (2010).

9. A. L. Belostotsky, A. S. Leonov, and A. V. Meleshko, Opt. Lett. 19, 856 (1994)

10. V.Z. Kolev, M.W. Duering, and B. Luther-Davies, Opt. Lett. 31, 2033-2035 (2006).

11. R.W. Boyd, Nonlinear Optics, Acad. Press, Boston, 1992. 


\section{Informational Fourth Page}

In this section, please provide full versions of citations to assist reviewers and editors (OL publishes a short form of citations) or any other information that would aid the peer-review process.

\section{References}

1. G. Cerullo and S. De Silvestri, 'Ultrafast optical parametric amplifiers', Rev. Sci. Instrum. 74, 1 (2003).

2. R. Butkus, R. Danielius, A. Dubietis, A. Piskarskas, and A. Stabinis, 'Progress in chirped pulse optical parametric amplifiers', Appl. Phys. B 79, 693 (2004).

3. P. Kuo, K. Vodopyanov, M. Fejer, D. Simanovskii, X. $\mathrm{Yu}$, J. Harris, D. Bliss, and D. Weyburne, 'Optical parametric generation of a mid-infrared continuum in orientation-patterned GaAs', Opt. Lett. 31, 71 (2006).

4. M. Tiihonen, V. Pasiskevicius, A. Fragemann, C. Canalias, and F. Laurell, 'Ultrabroad gain in an optical parametric generator with periodically poled KTiOPO $_{4}^{\prime}$, Appl. Phys. B 85, 73 (2006).

5. O. Prakash, H. H. Lim, B. J. Kim, K. Pandiyan, M. Cha, and B. Rhee, 'Collinear broadband optical parametric generation in periodically poled lithium niobate crystals by group velocity matching,' Appl. Phys. B 92, 535 (2008).

6. M. Levenius, V. Pasiskevicius, F. Laurell, and K. Gallo, 'Ultra-broadband optical parametric generation in periodically poled stoichiometric LiTaOs, Opt. Express 19, 4121 (2011).

7. G.I. Stegeman, $\mathbf{X}^{(2)}$ cascading: nonlinear phase shifts', Quantum Semiclass. Opt. 9, 139 (1997).

8. M. Conforti, F. Baronio, C. De Angelis, 'Nonlinear envelope equation for broadband optical pulses in quadratic media', Phys. Rev. A 81, 053841 (2010).

9. A. L. Belostotsky, A. S. Leonov, and A. V. Meleshko, 'Nonlinear phase change in type II second-harmonic generation under exact phase-matched conditions', Opt. Lett. 19, 856 (1994)

10. V.Z. Kolev, M.W. Duering, and B. Luther-Davies, 'Corrections to refractive index data of stoichiometric lithium tantalate in the 5-6 $4 \mathrm{~m}$ range', Opt. Lett. 31, 2033-2035 (2006).

11. R.W. Boyd, Nonlinear Optics, Acad. Press, Boston, 1992. 